\title{
IMPROPER MULTIPLE INTEGRALS OVER ITERABLE FIELDS*
}

\section{J. K. LAMOND}

\section{Introduction.}

LYTLE $†$ has shown that the fundamental relation

$$
\int_{\mathfrak{A}} f \leqq \int_{\mathfrak{B}} \int_{\mathfrak{G}}^{\cdot} f \leqq \overline{\int_{\mathfrak{B}}} \bar{\int}_{\mathfrak{E}} f \leqq \bar{\int}_{\mathfrak{A}} f,
$$

where the integrals are proper integrals, holds for a class of fields which he calls iterable (defined later in $\S 1$ ). In his Lectures, $\ddagger$ vol. II, Professor PIERPONT has given a new definition of an improper multiple integral (definition given later in $\S 1$ ). Considering functions defined, in general, over iterable fields and using Professor Pierpont's definition, I have shown that, under certain conditions, the above fundamental inequalities hold for a very general class of functions, some of which may have points of infinite discontinuity at an everywhere dense set in $\mathfrak{A}$, the field of integration.

For simplicity the proofs are given for functions of two independent variables. The methods of proof, however, are perfectly general and may be extended readily to any number of independent variables.

\section{§1. Preliminary Definitions. Uniform and Regular Convergence.}

Let $f\left(x_{1}, \cdots, x_{n}\right)$ be a function, defined over a limited field $\mathfrak{A}$, and let it have infinite discontinuities at the points of a set $\mathfrak{\Im}$, which may or may not be of zero content. The following is Pierpont's definition of the improper multiple integral of $f\left(x_{1}, \cdots, x_{n}\right)$ over $\mathfrak{A}$.

Let $\alpha \geqq 0$ and $\beta \geqq 0$ be two numbers, chosen at pleasure. Let $\mathfrak{A}_{\alpha \beta}$ be those points of $\mathfrak{A}$ at which

$$
-\alpha \leqq f\left(x_{1}, \cdots, x_{n}\right) \leqq \beta
$$

* Presented to the Society, April 27, 1912.

† Proper Multiple Integrals over Iterable Fields, Transactions of the American Mathematical Society, vol. 11 (1910), p. 25.

$\ddagger$ Lectures on the Theory of Functions of Real Variables, Ginn \& Co. In two volumes. These will be referred to as Lectures. 
and let $\mathfrak{A}_{a \beta}^{\prime}$ be the remaining points of $\mathfrak{A}$. If

$$
\lim _{a, \beta=\infty} \int_{\Phi_{\alpha \beta}} f
$$

exists, finite or infinite, it is defined to be the upper integral of $f\left(x_{1}, \cdots, x_{n}\right)$ over $\mathfrak{A}$ and is written

$$
\overline{\int_{x}} f .
$$

The lower integral of $f\left(x_{1}, \cdots, x_{n}\right)$ is similarly defined and written. When the upper and lower integrals of $f\left(x_{1}, \cdots, x_{n}\right)$ over $\mathfrak{A}$ are equal we denote their common values by

$$
\int_{\mathfrak{x}} f
$$

and say that $f\left(x_{1}, \cdots, x_{n}\right)$ is integrable in $\mathfrak{A}$.

Let now $\mathfrak{A}$ be a limited point aggregate in two dimensions. Let $\mathfrak{B}$ be the projection of $\mathfrak{A}$ on the $X$ axis and through each point $x$ of $\mathfrak{B}$ let an ordinate be erected. Each ordinate cuts a section $\mathfrak{E}(x)$ out of $\mathfrak{A}$. When no confusion can arise I denote this section simply by $\mathfrak{C}$. It is convenient to use the notation $\mathfrak{A}=\mathfrak{B} \cdot \mathfrak{C}$.

For the definition of the upper content of a set of points see Lectures, vol. I. The notation there employed will be used here.

According to LyTLE's definition* if the integral of the upper content of $\mathfrak{C}$ over $\mathfrak{B}$ exists and is equal to the upper content of $\mathfrak{A}$, that is, if

$$
\int_{\mathfrak{s}} \overline{\mathfrak{E}}=\overline{\mathfrak{A}},
$$

then $\mathfrak{A}$ is said to be iterable, modulo $\mathfrak{Y}$.

Let $\rho \geqq 0$ and $\sigma \geqq 0$ be two numbers chosen at pleasure. Let $\mathfrak{B}_{\rho \sigma}$ be those points of $\mathfrak{B}$ at which

$$
-\rho \leqq \overline{\int_{\mathbb{E}}} f \leqq \sigma
$$

If

$$
\lim _{\rho, \sigma=\infty} \overline{\int_{x_{\rho \sigma}}} \bar{\int}_{\dot{s}} f
$$

exists, finite or infinite, it is defined to be the upper integral of the integral in (2) over $\mathfrak{B}$, and is written

$$
\int_{\mathfrak{a}}^{-} \int_{\mathfrak{s}}^{-} f
$$

\footnotetext{
${ }^{*}$ Loc. cit.
} 
The lower, lower iterated integral, and the iterated integral are similarly defined.

It is evident that $f$ may be defined at the points of $\mathfrak{A}$ and yet the integral in (2) may not be defined at some or at any of the points of $\mathfrak{B}$. In this paper I shall restrict myself to the consideration of functions such that the integral in (2) shall exist and be finite, although not necessarily limited, except possibly at the points of a discrete set, at which points we may assign an arbitrary value to this integral without affecting the value of the iterated integral (see Lectures vol. II, § 60).

In what follows if an upper integral, a lower integral, or an integral is said to exist or to converge, I mean that it exists and is finite unless otherwise stated.

Let us suppose that the integral in (2) exists for each point $x$ in $\mathscr{B}$. Then if

$$
\epsilon>0, \quad \alpha_{0}, \beta_{0}, \quad\left|\int_{\mathfrak{G}_{a \beta}} f-\bar{\int}_{\mathfrak{G}} f\right|<\epsilon, \quad \alpha \geqq \alpha_{0}, \beta \geqq \beta_{0}, *
$$

and for each and every $x$ and $\mathfrak{B}$, we say that the integral in (2) converges uniformly in $\mathfrak{B}$.

Let $b$ be that partial set of $\mathfrak{B}$ such that the integral in (2) converges uniformly in $\mathfrak{B}-\mathfrak{b}$ and converges for each $x$ in $b$ except possibly at the points of a discrete set. Let $b_{\rho \sigma}$ be those points of $b$ at which relation (2) holds. If $\mathfrak{D}_{\rho \sigma}$ is discrete for each $\rho \geqq 0, \sigma \geqq 0$, and in addition $\overline{\mathfrak{P}}_{\rho \sigma}=\overline{\mathfrak{B}}$ for each $\rho \geqq \rho_{0}, \sigma \geqq \sigma_{0}, \rho_{0}, \sigma_{0}$ sufficiently large, we say that the integral in (2) converges regularly in $\mathfrak{B}$. If $D_{\rho \sigma}$ is discrete and $\overline{\mathfrak{B}}_{\rho \sigma}<\overline{\mathfrak{B}}$ for each $\rho \geqq 0, \sigma \geqq 0$, we say that the integral in (2) converges semi-regularly in $\mathfrak{B}$. If the integral in (2) converges regularly, or semi-regularly, in $\mathfrak{B}$, it follows at once, from Lectures, vol. II, $\S 360$, that the set $b$ is of measure zero.

Similar definitions may be given using lower integrals or integrals.

\section{§2. Improper Integrals.}

Theorem 1. Let

$$
\int_{\mathbb{G}}^{\bar{s}} f
$$

converge uniformly in $\mathfrak{B}$. Then (1) is limited in $\mathfrak{B}$ and the upper integral of (1) over $\mathfrak{B}$ exists.

For by the definition of uniform convergence, choosing $\epsilon>0$, small at

* This set of inequalities is written in accordance with Pierpont's notations. It means; for every positive $\epsilon$ there exists a pair of numbers, $\alpha_{0}$ and $\beta_{0}$, such that

for any $\alpha \geqq \alpha_{0}$, and for any $\beta \geqq \beta_{0}$.

$$
\left|\bar{\int}_{\mathfrak{C}_{a \beta}} f-\bar{\int}_{\mathfrak{E}} f\right|<\epsilon
$$


pleasure, there exists a fixed pair of numbers $\alpha, \beta$ such that

$$
\left|\bar{\int}_{\mathfrak{\epsilon}} f\right|<\left|\int_{\tilde{\epsilon}_{\alpha \beta}} f\right|+\epsilon
$$

for each and every $x$ in $\mathfrak{B}$. But

$$
\left|\bar{\int}_{\mathfrak{E}_{a \beta}} f\right| \leqq \mu \bar{B},
$$

where $B$ is the projection of $\mathfrak{A}$ on the $Y$ axis and $\mu=\operatorname{Max}(\alpha, \beta)$. Hence

$$
\left|\overline{\int_{\mathfrak{E}}} f\right|<\mu \bar{B}+\epsilon<M
$$

for each and every $x$ in $\mathfrak{B}$.

TheOREM 2. Let the upper integral of $f$ over $\mathbb{E}$ converge regularly, or semiregularly, in $\mathfrak{B}$. Then

exists.

$$
\overline{\int_{B}} \overline{\int_{\mathbb{E}}} f
$$

Let $F=0$ on sections corresponding to $b$,

$=f$ on sections corresponding to $\mathfrak{B}-\mathfrak{b}$.

Then for any $\rho, \sigma$

$$
\bar{\int}_{\mathfrak{x}_{\rho \sigma}} \overline{\int_{\mathfrak{G}}} f=\bar{\int}_{\mathfrak{x}_{\rho \sigma}} \overline{\int_{\mathfrak{E}}} F,
$$

since for points of $\mathfrak{F}_{\rho \sigma}$ the upper integral of $f$ over $\mathbb{C}$ differs from the upper integral of $F$ over $\mathbb{C}$ at only a discrete set $\delta_{\rho \sigma}$. Let now $\rho, \sigma \doteq \infty$. The upper integral of $F$ over $\mathfrak{C}$ converges uniformly in $\mathfrak{B}$. Hence, by Theorem 1 . the limit on the right of (1) exists. Hence the limit on the left exists.

Theorem 3. Let $f$ be limited in $\mathfrak{A}$ and let $\mathfrak{A}_{1}$ be a partial aggregate of $\mathfrak{A}$. Let, $1^{\circ}, f=0$ in $\mathfrak{A}-\mathfrak{A}_{1}$, or, $2^{\circ}$, let $\overline{\mathfrak{A}}_{1}=\overline{\mathfrak{A}}$. In either case

$$
\int_{\mathscr{x}} f \leqq \int_{\mathscr{x}_{1}} f, \quad \bar{\int}_{\mathbf{x}_{1}} f \leqq \bar{\int}_{\mathfrak{x}} f .
$$

Effect a cubical division of space and let the cells containing points of both $\mathfrak{A}$ and $\mathfrak{A}_{1}$ be $d_{1}, \cdots, d_{n}$. Let the remaining cells which contain points of $\mathfrak{A}$ be $d_{1}^{\prime}, \cdots, d_{m}^{\prime}$. If condition $1^{\circ}$ is fulfilled, $f=0$ in cells $d_{i}^{\prime}$. If condition $2^{\circ}$ is fulfilled, $\Sigma d_{i}^{\prime}$ is small at pleasure. In any cell $d_{i}$, if either condition $1^{\circ}$ or condition $2^{\circ}$ is fulfilled, $\operatorname{Max} f$ at points of $\mathfrak{A}_{1} \leqq \operatorname{Max} f$ at points of $\mathfrak{A}$ and $\operatorname{Min} f$ at points of $\mathfrak{U} \leqq \operatorname{Min} f$ at points of $\mathfrak{A}_{1}$. Hence the inequalities (1) follow.

THEOREM 4. Let the upper integral of $f$ over $\mathfrak{E}$ converge regularly in $\mathfrak{B}$. Then

$$
\overline{\int_{\S}} \overline{\int_{\mathfrak{E}}} f \leqq \lim _{a, \beta=\infty} \bar{\int}_{\mathscr{B}} \bar{\int}_{\mathfrak{G}_{\alpha \beta}} f .
$$


By the definition of regular convergence, and Theorem 1, there exists a pair of numbers $\rho_{0}, \sigma_{0}$ such that

$$
\overline{\mathfrak{B}}_{\rho \sigma}=\overline{\mathfrak{B}},
$$

$$
\mathfrak{B}-\mathfrak{b}=\mathfrak{B}_{\rho \sigma}-\mathfrak{b}_{\rho \sigma}, \quad \text { for } \quad \rho \geqq \rho_{0}, \quad \sigma \geqq \sigma_{0} .
$$

Since $b_{\rho \sigma}$ is discrete, it follows from (3) that

$$
\overline{\int_{\mathfrak{g}-\mathrm{b}}} \bar{\int}_{\mathfrak{E}} f=\bar{\int}_{\mathfrak{x}_{\rho \sigma}} \int_{\mathfrak{G}} f .
$$

Passing to the limit $\rho, \sigma=\infty$ in this relation we have, by Theorem 2,

$$
\overline{\int_{\mathfrak{B}-b}} \overline{\int_{\mathfrak{E}}} f=\overline{\int_{\mathfrak{g}}} \int_{\mathfrak{E}}^{\bar{a}} f .
$$

Relations (2) and (3) give $\overline{\mathfrak{P}}=\operatorname{Upper}$ Cont. $(\mathfrak{B}-\mathfrak{b})$. Hence, by Theorem 3 , for any $\alpha, \beta$, arbitrary but fixed,

$$
\overline{\int_{y-b}} \overline{\int_{\mathfrak{E}_{\alpha \beta}}} f \leqq \overline{\int_{\mathscr{B}}} \overline{\int_{\mathfrak{G}_{\alpha \beta}}} f .
$$

By Lectures, vol. II, $\S 150$,



This with the limit of (5) gives

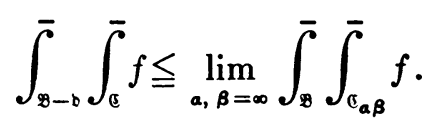

This relation with (4) gives (1).

Corollary 1. Let $f \geqq 0$ and let the upper integral of $f$ over $\mathbb{\complement}$ converge semiregularly in $\mathfrak{B}$. Then

$$
\overline{\int_{\mathfrak{B}}} \overline{\int_{\mathfrak{E}}} f \leqq \lim _{\alpha, \beta=\infty} \bar{\int}_{\mathfrak{B}} \overline{\int_{\mathfrak{E}_{\alpha \beta}} f} .
$$

Since the upper integral of $f$ over $\mathbb{C}$ converges semi-regularly in $\mathfrak{B}$, we have relation (3) and hence equation (4). Since $f \geqq 0$, the inequality (5) is true. The limit of this with (4) gives the corollary.

In connection with relation (4) it is well to notice that if the upper integral of $f$ over $\mathfrak{C}$ converges regulariy in $\mathfrak{B}$, we may disregard the set $\mathfrak{b}$, of measure zero, in computing

$$
\overline{\int_{9}} \int_{\mathfrak{G}} f .
$$

That the inequality sign is necessary in (1) is shown by the following example. 
Example 1. Let $\mathfrak{A}$ be the unit square.

Let $f=n$ at the points ${ }^{*} x=m / n, y$ rational, in $\mathfrak{A}$,

$=2$ at the points $x=m / n, y$ irrational, in $\mathfrak{A}$,

$=1$ at the remaining points in $\mathfrak{A}$.

Let $\beta \geqq 2$ be arbitrary but fixed. For $x$ irrational

For $x$ rational

$$
\overline{\int_{\mathfrak{E}}} f=1, \quad \bar{\int}_{\mathfrak{G}_{\beta}} f=1,
$$

$$
\overline{\int_{\mathfrak{E}}} f=n, \quad \bar{\int}_{\mathfrak{a}_{\boldsymbol{\beta}}} f=2
$$

except for a discrete set in the case of the latter integral. Hence

$$
\overline{\int_{B}} \int_{\mathfrak{E}} f=1, \quad \lim _{\boldsymbol{\beta}=\infty} \bar{\int}_{\mathfrak{B}}^{\bar{\delta}} \int_{\mathfrak{E}_{\boldsymbol{\beta}}} f=2 .
$$

It is convenient to introduce an auxiliary function $\phi_{\alpha \beta}$ defined as follows: $\phi_{\alpha \beta}$ shall be equal to $f$ at the points of $\mathfrak{A}_{\alpha \beta}$ and equal to zero at the points of $\mathfrak{U}_{a \beta}^{\prime}$.

Theorem 5. Let both the integrals

exist. Let $\overline{\mathfrak{\Im}}=0$. Then

$$
\int_{x}^{\bar{x}} f
$$

$$
\lim _{a, \beta=\infty} \int_{\mathfrak{a}}^{\bar{a}} \phi_{a \beta}=\int_{\mathfrak{a}}^{\bar{a}} f .
$$

Since $\overline{\mathfrak{J}}=0, \overline{\mathfrak{A}}_{\alpha \beta}^{\prime}$ may be made small at pleasure by taking $\alpha, \beta$ sufficiently large. Let $\mathfrak{U}_{\alpha \beta}^{\prime}$ be enclosed in a set of cells $d_{1}, \cdots, d_{n}$ of norm $d$. Then

$$
\epsilon>0, \quad \alpha_{0}, \beta_{0}, d_{0}, \quad \Sigma d_{i}<\epsilon, \quad \alpha \geqq \alpha_{0}, \beta \geqq \beta_{0}, \quad d \leqq d_{0} .
$$

Let these last quantities be arbitrary but fixed. Let $\mathfrak{A}_{1}$ be points of $\mathfrak{A}$ lying in cells $d_{i}$, and $\mathfrak{A}_{2}$ be the remaining points of $\mathfrak{A}$. Since $\mathfrak{A}_{1}$ and $\mathfrak{A}_{2}$ are unmixed, by Lectures, vol. II, $\S 46$,

$$
\overline{\int_{\mathfrak{x}}} f=\overline{\int_{\mathfrak{x}_{1}}} f+\bar{\int}_{\mathfrak{x}_{2}} f, \quad \overline{\int_{\mathfrak{x}}} \phi_{a \beta}=\bar{\int}_{\mathfrak{x}_{1}} \phi_{a \beta}+\bar{\int}_{\mathfrak{x}_{2}} \phi_{a \beta} .
$$

Since $f=\phi_{a \beta}$ at points of $\mathfrak{A}_{2}$,

$$
\int_{\mathfrak{x}_{2}}^{\bar{a}} f=\bar{\int}_{\mathfrak{x}_{2}} \phi_{a \beta} .
$$

* All fractions, here and elsewhere, are supposed to be irreducible.

$\dagger$ In the case of a function of one sign, the notation may be simplified by dropping either the $\alpha$ or the $\beta$ as the case may be.

$\ddagger$ For the significance of this system of inequalities cf. footnote on page 436 . 
Hence, subtracting the equations in relation (2) and using Lectures, vol. II, $\S \S 51$ and 48,3 we have

(3) $\left|\overline{\int_{x}} f-\overline{\int_{x}} \phi_{a \beta}\right|=\left|\overline{\int_{x_{1}}} f-\bar{\int}_{x_{1}} \phi_{a \beta}\right| \leqq\left|\bar{\int}_{x_{1}} f^{\prime}+\right| \bar{\int}_{x_{1}} \phi_{a \beta}\left|\leqq \bar{\int}\right| f\left|+\bar{\int}_{x_{1}}\right| \phi_{a \beta} \mid$.

In $\mathfrak{A}_{1},\left|\phi_{a \beta}\right|$ equals $|f|$ at some points and equals zero at the remaining points. From this fact and Lectures, vol. II, § 48, 3 and $\S 53$

$$
\overline{\int_{\mathbf{x}_{1}}}\left|\phi_{a \beta}\right| \leqq \bar{\int}_{\mathbf{x}_{1}}|f|<\frac{1}{2} \eta
$$

where $\eta$ may be taken small at pleasure by taking $\epsilon$ sufficiently small. Relations (3) and (4) give

$$
\left|\int_{*} f-\int_{*} \phi_{a \beta}\right|<\eta \text {. }
$$

Now $\epsilon \doteq 0$ and $\alpha, \beta \doteq \infty$ when $\eta \doteq 0$. Hence we get (1) by passing to the limit $\eta=0$ in (5).

Theorems similar to Theorems $1,2,4$, and 5 are readily proved for lower integrals.

ThEoREm 6. Let $\mathfrak{A}=\mathfrak{B}$. $\mathfrak{C}$ be iterable modulo $\mathfrak{B}$. Let both the integrals

$$
\tilde{\int}_{-1} f
$$

exist. Let $\bar{\Im}=0$. Let both the integrals

$$
\bar{\int}_{\mathbb{G}} f
$$

converge uniformly or regularly in $\mathfrak{B}$. Then

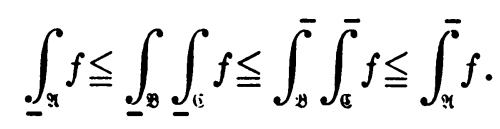

Let $\alpha, \beta$ be arbitrary but fixed, and let $\phi_{a \beta}$ be the limited auxiliary function already defined. In any section $\mathfrak{C}, \mathfrak{C}_{a \beta}<\mathfrak{C}$ and $\phi_{\alpha \beta}$ equals $f$ at points of $\mathfrak{C}_{\alpha \beta}$ and equals zero at points of $\mathbb{C}_{a \beta}^{\prime}$. Hence it follows from Theorem 3 that

and hence that

$$
\int_{\mathbb{\sigma}} \phi_{\alpha \beta} \leqq \int_{\mathbb{G}_{\alpha \beta}} f \leqq \int_{\mathbb{G}_{\alpha \beta}} f \leqq \int_{\mathbb{G}} \phi_{\alpha \beta},
$$

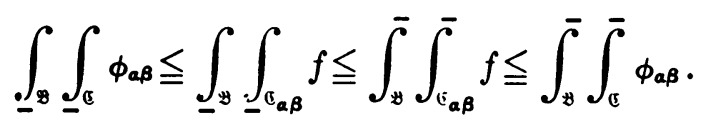


By Lytle's theorem 14 (loc. cit., p. 35) we have

$$
\int_{\mathfrak{X}} \phi_{a \beta} \leqq \int_{\mathfrak{B}} \int_{\mathfrak{C}} \phi_{a \beta} \leqq \int_{\mathfrak{B}} \int_{\mathfrak{C}}^{\bar{d}} \phi_{a \beta} \leqq \bar{\int}_{\mathfrak{X}} \phi_{a \beta},
$$

and this with (3) gives

$$
\int_{\mathfrak{Q}} \phi_{a \beta} \leqq \int_{\mathscr{B}} \int_{\mathfrak{G}_{\alpha \beta}} f \leqq \int_{\mathcal{B}} \int_{\mathfrak{E}_{\alpha \beta}} f \leqq \int_{\mathfrak{M}} \phi_{a \beta} .
$$

Passing to the limit $\alpha, \beta=\infty$ in this last relation, and using Theorem 5 and Lectures, vol. II, $\S 150$, in case that the integrals (1) converge uniformly in $\mathfrak{B}$, and Theorems 4 and 5 , in case the integrals (1) converge regularly in $\mathfrak{B}$, we have*

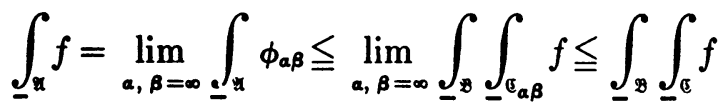

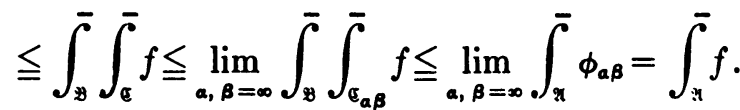

This gives relation (2).

CoRollary 1. Let the conditions of Theorem 6 be satisfied and in addition let $f$ be integrable in $\mathfrak{A}$. Then

$$
\int_{\mathfrak{x}} f=\int_{\mathbb{*}^{*}} \int_{\mathbb{B}} f=\bar{\int}_{\mathbb{H}}^{\overline{0}} \overline{\int_{\mathfrak{G}}} f .
$$

If $f$ is also integrable in each $\mathfrak{C}$ in $\mathfrak{B}$

$$
\int_{\mathfrak{M}} f=\int_{\mathbb{y}} \int_{\mathfrak{B}} f
$$

Let $B$ be the projection of $\mathfrak{A}$ on the $Y$ axis and $C$ the corresponding section.

Corollary 2. Let $\mathfrak{A}=\mathfrak{B} \cdot \mathfrak{C}=B \cdot C$ be iterable modulis $\mathfrak{B}, B$. Let $f$ be integrable in $\mathfrak{A}$.

Let the integrals (1) converge uniformly or regularly in $\mathfrak{B}$ and the integrals

$$
\int_{c}^{\bar{c}} f
$$

converge uniformly or regularly in $B$. Then

$$
\int_{\mathfrak{n}} f=\bar{\int}_{\sharp}^{\bar{J}} \overline{\int_{\mathfrak{G}}}=\overline{\int_{B}} \bar{\int}_{C} f .
$$

Example 2. Let $\mathfrak{A}$ be the points in a unit square whose abscissas are rational.

* In case the integrals (1) converge uniformly in $\mathfrak{B}$ the sccond and fourth inequality signs may be left out of this relation. 
Let $f$ be defined over $\mathfrak{A}$ as follows:

$$
\begin{aligned}
& f=n(-1)^{n} \text { at the points } x=1 / n, \quad 0 \leqq y \leqq 1, \\
& =1 \quad \text { at the points } x \neq 1 / n, \quad y \text { rational, } \\
& =2 \quad \text { at the points } x \neq 1 / n, \quad y \text { irrational. }
\end{aligned}
$$

Then we have

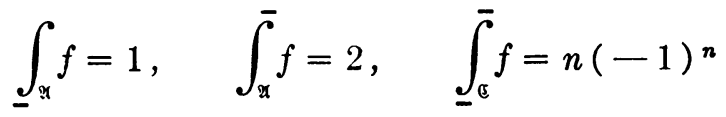

for $x=1 / n$. For $x$ rational but not equal to $1 / n$,

Hence

$$
\int_{\mathfrak{G}} f=1, \quad \int_{\mathfrak{G}}^{\bar{a}} f=2 .
$$

$$
\int_{\mathfrak{B}} \int_{\mathfrak{G}} f=1, \quad \int_{\mathfrak{B}} \int_{\mathfrak{G}} f=2 .
$$

In connection with this example we may note the following points. The integrals over $\mathfrak{C}$ converge regularly in $\mathfrak{B} ; \overline{\mathfrak{J}}=0 ; \mathfrak{A}$ does not possess content; $\mathfrak{B}$ neither possesses content nor is complete; and the function $f$ is limited in neither direction.

Example 3. Let $\mathfrak{A}$ be a unit square and let $f$ be defined over $\mathfrak{A}$ as follows:

$$
\begin{aligned}
f & =n \text { at points } x=m / n, \quad 0 \leqq y \leqq 1 / n, \\
& =1 \text { elsewhere in } \mathfrak{A} .
\end{aligned}
$$

Then

$$
\bar{\int}_{\underline{n}}^{\overline{0}} f=1, \quad \int_{G}^{\bar{c}} f=n \int_{0}^{1 / n} d y+\int_{1 / n}^{1} d y=2-1 / n
$$

for $x=m / n$. Elsewhere in $\mathfrak{B}$

Hence

$$
\overline{\int_{\circledast}} f=1
$$

$$
\int_{\mathfrak{D}} \int_{\mathfrak{E}} f=1, \quad \int_{\mathfrak{B}}^{\bar{a}} \int_{\mathfrak{E}}^{\bar{\top}} f=2 .
$$

We see that the fundamental relation breaks down. However

$$
\bar{\int}_{\mathfrak{c}}^{\bar{c}} f
$$

do not converge either uniformly or regularly in $\mathfrak{B}$.

THeOREm 7. Let $f$ be of one sign or zero and defined over $\mathfrak{A}=\mathfrak{B} \cdot \mathbb{E}$ which 
may or may not be iterable modulo $\mathfrak{B}$. Let $\bar{\Im} \geqq 0$. Then, if $f \geqq 0$ and the upper integral of $f$ over $\mathfrak{A}$ exists and the upper integral of $f$ over $\mathfrak{E}$ converges uniformly, regularly, or semi-regularly in $\mathfrak{B}$,

$$
\overline{\int_{B}} \overline{\int_{\mathfrak{E}}} f \leqq \overline{\int_{\mathfrak{B}}} f .
$$

If $f \leqq 0$, and the lower integral of $f$ over $\mathfrak{A}$ exists and the lower integral of $f$ over $\mathfrak{C}$ converges uniformly, regularly, or semi-regularly in $\mathfrak{B}$, then

$$
\int_{-x} f \leqq \int_{-y} \int_{\mathbb{E}} f
$$

Let us prove (1); (2) is proved in a similar manner. Let $\phi_{\beta}$ be the auxiliary function already defined. As in the latter part of equation (3), Theorem 6,

$$
\bar{\int} \int_{\mathbb{B}} f \leqq \overline{\int_{B}} \overline{\int_{\mathfrak{S}}} \phi_{\beta} \text {. }
$$

By two theorems proved by Richardson*

$$
\overline{\int_{\mathfrak{B}}} \bar{\int}_{\mathfrak{Q}} \phi_{\beta} \leqq \int_{\mathfrak{Q}} \phi_{\beta}=\bar{\int}_{\boldsymbol{x}_{B}} f
$$

This with relation (3) gives

$$
\bar{\int} \int_{\mathbb{B}} f \leqq \int_{\mathfrak{Q}_{\beta}} f .
$$

Passing to the limit as in equation (4), Theorem 6, we have (1).

Example 4. Let $f$ be a function defined over a set of points $\mathfrak{A}$, lying in a unit square, as follows:

$$
\begin{aligned}
f= & n \cdot q \text { at the points } x=m / n, \quad y=1 / q, \\
= & n \text { for } x \text { irrational, } \quad y=m / n, \\
= & 1 \text { for } x \text { irrational, } \quad y \text { irrational. } \\
& \bar{\int}_{\mathscr{N}} f=1, \quad \int_{\mathbb{*}} \underline{\int}_{\mathbb{G}} f=0, \quad \bar{\int} \bar{\int}_{\mathbb{G}} f=1 .
\end{aligned}
$$

Here $\overline{\mathfrak{\Im}}>0$. $\mathfrak{A}$ is not iterable since $\overline{\mathbb{C}}(x)$ equals zero for $x$ rational and equals 1 for $x$ irrational. Hence

does not exist.

$$
\int_{\mathbb{y}} \overline{\mathfrak{E}}(x)
$$

* The Integration of a Sequence of Functions and Its Application to Iterated Integrals, Tr an 8actions of the American Mathematical Society, vol. 9 (1908), p. 347. 
ThEoRem 8. Let $\mathfrak{A}_{\alpha \beta}=\mathfrak{B} \cdot \mathfrak{C}_{\alpha \beta}$ be iterable modulo $\mathfrak{B}$ for any $\alpha \geqq \alpha_{0}$, $\beta \geqq \beta_{0} ; \alpha_{0}, \beta_{0}$ sufficiently large. Let $\bar{\Im} \geqq 0$. Let both the integrals

exist. Let both the integrals

$$
\bar{\int}_{\mathfrak{x}} f
$$

$$
\tilde{\int}_{\sigma} f
$$

converge uniformly or regularly in $\mathfrak{B}$. Then

(1)

$$
\int_{\mathscr{A}} f \leqq \int_{\mathfrak{Y}} \int_{\mathbb{E}} f \leqq \int_{\mathfrak{Y}}^{\bar{T}} \int_{\mathfrak{C}} f \leqq \int_{\mathscr{Y}} f .
$$

For by Lytle's Theorem 14 (loc. cit., p. 35), for $a \geqq \alpha_{0}, \beta \geqq \beta_{0}$,

$$
\int_{\mathbb{*}_{\alpha \beta}} f \leqq \int_{\mathfrak{y}} \int_{\mathfrak{E}_{\alpha \beta}} f \leqq \int_{\mathfrak{B}} \int_{\mathfrak{C}_{\alpha \beta}} f \leqq \bar{\int}_{\mathfrak{x}_{\alpha \beta}} f .
$$

Passing to the limit $\alpha, \beta=\infty$ in this relation as in equation (4) of Theorem 6 we get (1).

Example 5. Let $\mathfrak{A}$ be the points of a unit square whose abscissas are rational and let $f$ be defined over $\mathfrak{A}$ as follows:

$$
\begin{aligned}
f & =n \text { at the points } x=m / 2^{n}, \quad y \text { rational, } \\
& =1 \text { at the points } x=m / 2^{n}, \quad y \text { irrational, } \\
& =2 \text { at the remaining points of } \mathfrak{A} . \\
\int_{x} f & =1, \quad \int_{\mathfrak{x}} f=2, \quad \int_{*} \int_{\mathbb{N}} f=1, \quad \int_{\mathbb{N}} \bar{\int}_{\mathbb{G}} f=2 .
\end{aligned}
$$

WESLEYAN UNIVERSITY,

Middetown, Conn., A pril, 1912. 